\title{
List A Trial 5 Free Recall
}

National Cancer Institute

\section{Source}

National Cancer Institute. List A Trial 5 Free Recall. NCI Thesaurus. Code C120392.

The final free recall task from List A of the California Verbal Learning Test for Children. 